CARDIOVASCULAR MEDICINE

\title{
Image quality and diagnostic accuracy of non-invasive coronary imaging with 16 detector slice spiral computed tomography with $188 \mathrm{~ms}$ temporal resolution
}

\author{
A Kuettner, T Beck, T Drosch, K Kettering, M Heuschmid, C Burgstahler, C D Claussen, A F Kopp, \\ S Schroeder
}

Heart 2005;91:938-941. doi: 10.1136/hrt.2004.044735

See end of article for authors' affiliations

Correspondence to: Priv-Doz Dr Stephen Schröder, Department of Cardiology, EberhardKarls University

Tuebingen, Ottried-

Mueller Strasse 10, 72076

Tuebingen, Germany;

stephen.schroeder@med.

uni-tubingen.de

Accepted

17 September 2004
Objective: To evaluate image quality and clinical accuracy in detecting coronary artery lesions with a new multidetector spiral computed tomography (MDCT) generation with 16 detector slices and a temporal resolution of $188 \mathrm{~ms}$.

Methods: 124 consecutive patients scheduled for invasive coronary angiography (ICA) were additionally studied by MDCT (Sensation 16 Speed 4D). MDCTs were analysed with regard to image quality and presence of coronary artery lesions. The results were compared with ICA.

Results: 120 of 124 scans were successful. The image quality of all remaining 120 scans was sufficient (mean (SD) heart rate 64.2 (9.8) beats/min, range 43-95). The mean calcium mass was 167 (223) mg (range 0-1038). Thirteen coronary segments were evaluated for each patient (1560 segments in total). Image quality was graded as follows: excellent, 422 (27.1\%) segments; good, 540 (34.6\%) segments; moderate, 277 (17.7\%) segments; heavily calcified, 215 (13.8\%) segments; and blurred, 106 (6.8\%) segments. ICA detected 359 lesions with a diameter stenosis $>50 \%$ and MDCT detected 304 of 359 (85\%). Sensitivity, specificity, and positive and negative predictive values were $85 \%, 98 \%, 91 \%$, and $96 \%$, respectively. The correct clinical diagnosis (presence or absence of at least one stenosis $>50 \%$ ) was obtained for 110 of 120 (92\%) patients.

Conclusions: MDCT image quality can be further improved with 16 slices and faster gantry rotation time. These results in an unselected population underline the potential of MDCT to become a non-invasive diagnostic alternative, especially for the exclusion of coronary artery disease, in the near future.
M echanical multidetector spiral computed tomography (MDCT) systems have been available since 1999 for the non-invasive visualisation of human coronary arteries. Continuous technical innovations have led to a further stabilisation of image quality allowing a high diagnostic accuracy to rule out or detect significant coronary lesions in selected patients. Nieman et $a l^{1}$ and Ropers et $a l^{2}$ reported promising results in selected patients with clinical suspicion of coronary artery disease $(\mathrm{CAD})$ with the use of MDCT systems with $12 \times 0.75 \mathrm{~mm}$ cross sections and $420 \mathrm{~ms}$ rotation time.

In autumn 2003, the latest generation of 16 slice scanners was introduced. They featured 16 slices for cardiac imaging, together with an improved gantry rotation time $(16 \times 0.75 \mathrm{~mm}, 375 \mathrm{~ms})$.

The objective of our study was to evaluate the feasibility, image quality, and clinical accuracy in detecting coronary artery lesions with this scanner generation in an unselected patient population scheduled for invasive coronary angiography (ICA).

\section{METHODS}

\section{Study population}

We screened 195 consecutive patients scheduled for ICA because of suspected CAD or suspected progress of a known $\mathrm{CAD}$ for participation in the present study. All patients were listed for ICA because of typical chest pain or positive stress tests.

Seventeen (9\%) patients declined to participate, 47 (24\%) patients had contraindications, and for eight (4\%) patients no scan time was available. Of the 195 patients, 124 (64\%) were enrolled in this study. The local ethics committee had approved the study protocol and all patients gave informed consent. Exclusion criteria were defined as irregular heart rate, contraindications to iodinated contrast media, and increased serum creatinine concentrations $>133 \mu \mathrm{mol} / \mathrm{l}$. All patients underwent MDCT one day before ICA. Patients with heart rates $>65$ beats/min were given additional $\beta$ blockade.

\section{Scan protocol}

MDCT was performed with a 16 detector slice computed tomograph (Sensation 16 Speed 4D, Siemens Medical Solutions, Forchheim, Germany) with a newly developed tube (Straton, Siemens Medical Solutions), 370 ms rotation time, and 16 slices read out simultaneously in the cardiac mode. The scan protocol was as follows: native vessels were scanned without contrast medium to determine the total calcium burden of the coronary tree $(16 \times 1.5 \mathrm{~mm}$ collimation, table feed $3.8 \mathrm{~mm} /$ rotation, tube current $133 \mathrm{~mA}$ at $120 \mathrm{kV}$ ). To evaluate the circulation time, $20 \mathrm{ml}$ of contrast medium $(20 \mathrm{ml}$ at $4 \mathrm{ml} / \mathrm{s}, 400 \mathrm{mg}$ iodine $/ \mathrm{ml}$, Imeron 400; Altana Pharma, Constance, Germany) and a chaser bolus of $20 \mathrm{ml}$ saline were administered in an antecubital vein. The contrast enhanced scan of the coronary arteries was acquired with the following protocol: $80 \mathrm{ml}$ intravenous contrast agent followed by $20 \mathrm{ml}$ chaser bolus $(50 \mathrm{ml}$ at $4.0 \mathrm{ml} / \mathrm{s}$, then 30 or $50 \mathrm{ml}$ at $2.5 \mathrm{ml} / \mathrm{s}), 16 \times 0.75 \mathrm{~mm}$ collimation, table feed $3.8 \mathrm{~mm} /$ rotation, tube current $650 \mathrm{~mA}$ at $120 \mathrm{kV}$. Computed tomography started at the aortic root cranial to the coronary

Abbreviations: $C A D$, coronary artery disease; ICA, invasive coronary angiography; $M D C T$, multidetector spiral computed tomography 

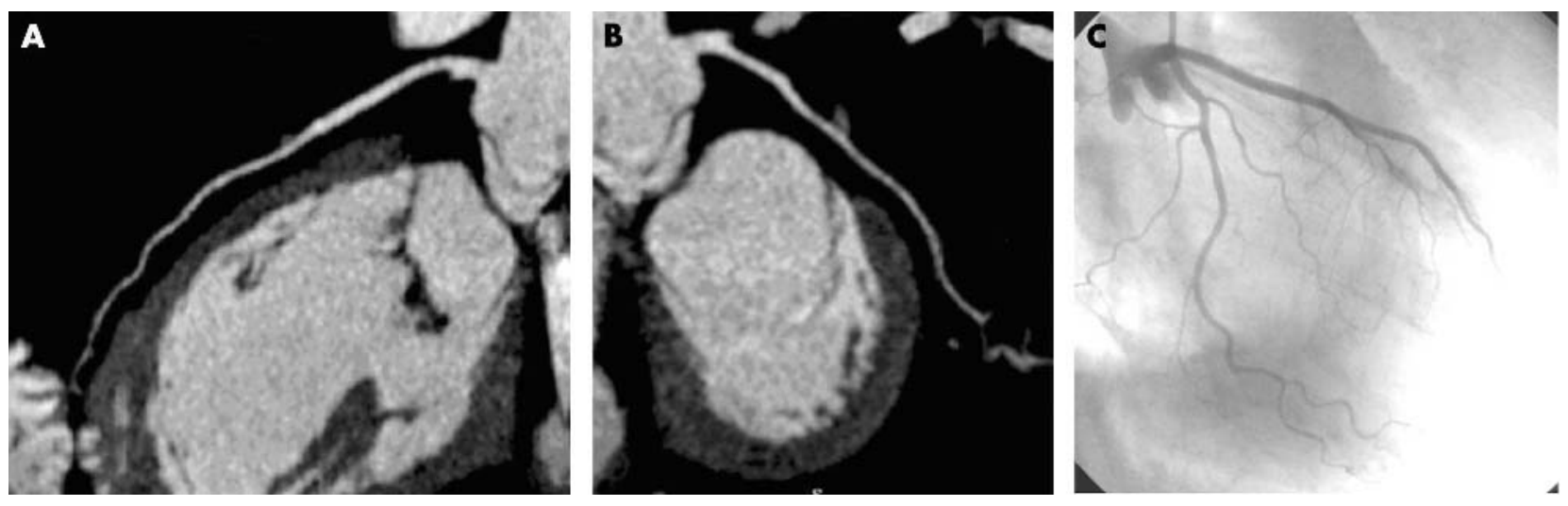

Figure 1 Exclusion of coronary artery disease in a patient. (A) Curved multiplanar reconstruction of the left anterior descending artery. (B) Curved multiplanar reconstruction of the left circumflex artery. (C) Corresponding coronary angiography.

ostia and stopped at the diaphragm caudally of all cardiac structures. ECG pulsing with reduced tube current during systole was recorded during all scans to minimise radiation exposure.

\section{Image reconstruction}

Axial images were reconstructed by the standard built-in ECG gated half scan reconstruction algorithm (temporal resolution $185 \mathrm{~ms}$, slice thickness $1.0 \mathrm{~mm}$, increment $0.5 \mathrm{~mm}$, kernel B $30 \mathrm{f}$ ). The reconstruction window was set to start at $60 \%$; in case of motion artefacts an additional test series reconstructing single slices in 5\% steps ranging from $20-75 \%$ relative to the RR interval was adopted. Examples of images are shown in figs 1 and 2 .

\section{MDCT image interpretation}

Two reviewers, blinded to the results of ICA and to all clinical information, evaluated the MDCTs in a joint reading. Data were analysed on an offline workstation for postprocessing (Leonardo, Siemens, Forchheim, Germany). Coronary calcifications were assessed on native scans and quantified by determining the total calcium mass expressed in $\mathrm{mg}$ of calcium hydroxyapatite.

Contrast enhanced MDCT angiograms were investigated by the use of axial slices and $4 \mathrm{~mm}$ thin slab maximum intensity projections in axial and double oblique orientation adapted to the specific anatomy of the vessel.

Image quality was classified as excellent (no motion artefacts present), good (minor motion artefacts present), moderate (substantial motion artefacts present but luminal assessment regarding significant stenosis still possible), heavily calcified (vessel lumen obscured by calcification), or blurred (no luminal assessment of significant stenosis possible).

The readers assessed significant lesions with a diameter stenosis $\geqslant 50 \%$. Results were documented separately for 13 coronary segments from each patient according to a modified American Heart Association classification with 13 segments (right coronary artery, segments 1-4; left main, segment 5; left anterior descending coronary artery, segments 6-10; left circumflex artery, segments 11-13).

The clinical diagnosis based on MDCT was considered to be correct if MDCT ruled out any significant lesion $>50 \%$ or MDCT detected correctly at least one significant lesion > 50\% that was detected by ICA.

\section{Quantitative coronary angiography}

ICA was performed with standard techniques one day after the MDCT. All angiograms were evaluated by one blinded independent observer by quantitative coronary analysis (Philips Medical Systems, Einthoven, the Netherlands). The projection with the most severe degree of diameter stenosis was used for evaluation. Lesions with a diameter stenosis $>50 \%$ were considered to be significant.

\section{Statistical analysis}

ICA was regarded as the reference standard for the detection of significant lesions. MDCT results were compared on a segmental basis, for each vessel and each patient. If a segment contained more than one lesion, the most severe lesion determined the diagnostic accuracy of the assessment. Categorical data were presented with absolute frequencies
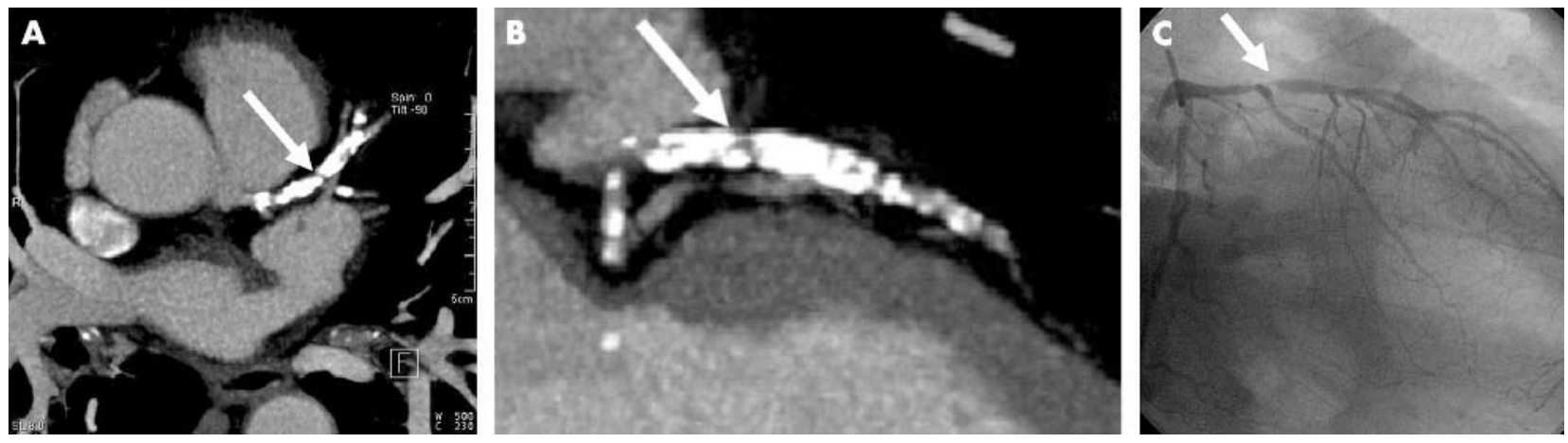

Figure 2 Patient with severe calcifications (140 mg calcium hydroxyapatite) in projection on the left anterior descending artery with a tight stenosis (arrows) between two calcified plaques immediately after the delivery of the first diagonal branch. (A) Axial multidetector spiral computed tomography (MDCT) image showing heavy calcifications. (B) Perpendicular angle. (C) Corresponding coronary angiography. 
Table 1 Number of coronary segments with diagnostic image quality

\begin{tabular}{llll}
\hline & ICA & MDCT & Evaluable \\
\hline RCA segments & & & \\
$\quad$ Total & 480 & 461 & $96 \%$ \\
1 & 120 & 120 & $100 \%$ \\
2 & 120 & 117 & $98 \%$ \\
3 & 120 & 114 & $95 \%$ \\
4 & 120 & 110 & $92 \%$ \\
LM segment & & & \\
5 & 120 & 118 & $98 \%$ \\
LAD segments & & & \\
Total & 600 & 539 & $90 \%$ \\
6 & 120 & 118 & $98 \%$ \\
7 & 120 & 119 & $99 \%$ \\
8 & 120 & 117 & $98 \%$ \\
9 & 120 & 100 & $83 \%$ \\
10 & 120 & 85 & $71 \%$ \\
LCx segments & & & \\
Total & 360 & 336 & $93 \%$ \\
11 & 120 & 116 & $97 \%$ \\
12 & 120 & 113 & $94 \%$ \\
13 & 120 & 107 & $89 \%$ \\
\hline
\end{tabular}

ICA, invasive coronary angiography; LAD, left anterior descending coronary artery; $L C x$, left circumflex artery; $L M$, left main artery; $M D C T$, multidetector spiral computed tomography; RCA, right coronary artery.

and percentages. Continuous variables are shown as mean (SD). Data were analysed with Prism 3.0 (GraphPad Software Inc, San Diego, California, USA).

\section{RESULTS}

\section{Patient population}

Of 195 consecutive patients scheduled for ICA, 124 (64\%) were suitable for MDCT and participation in our study. Clinical indication for ICA was suspicion of CAD in 80 of 124 (65\%) patients and suspicion of restenosis in 44 of 124 (35\%) patients. Fourteen patients had stented lesions which were excluded from analysis, and 13 patients had coronary artery bypass grafts. Patient characteristics were as follows: 83 were men $(67 \%)$, mean (SD) age was 64.1 (9.1) years, heart rate was 64.2 (9.8) beats/min (range 43-95, mean calcium mass was 167 (223) mg (range 0-1038), and body mass index was $28.2(3.8) \mathrm{kg} / \mathrm{m}^{2}$ (range 20-38). Cardiovascular risk factors were distributed as follows: hyperlipoproteinaemia, 97 of 124 (78\%); hypertension, 90 of 124 (73\%); smoking, 42 of 124
(34\%); family history, 25 of 124 (20\%); and diabetes, 22 of $124(18 \%)$.

\section{Image quality}

Additional $\beta$ blockade was given to 63 of 124 (51\%) patients before MDCT because of heart rates $>65$ beats/min at inclusion. Of $124(97 \%)$ scans, 120 were successful (two incomplete respiratory stops, one ECG defect, one extravasation of contrast medium). All remaining 120 scans had sufficient image quality. In total, 1560 segments ( 120 patients $\times 13$ segments) were evaluated. Image quality was graded as follows: excellent, 422 (27.1\%) segments; good, 540 (34.6\%) segments; moderate, 277 (17.7\%) segments; heavily calcified, $215(13.8 \%)$ segments; and blurred, 106 (6.8\%) segments. The entire coronary tree was visualisable with diagnostic image quality in 60 of $120(50 \%)$ patients. Table 1 summarises the results of the segmental and per vessel analysis.

\section{Bypass imaging}

Forty one of 43 bypass grafts (95\%) were analysable by MDCT in 13 patients after coronary artery bypass grafting (11 arterial grafts, 32 venous grafts). In ICA, 16 of 43 (37\%) grafts were significantly stenosed $(>70 \%)$, and 16 of them were correctly diagnosed by MDCT (sensitivity 100\%). One graft had a $50 \%$ anastomosis stenosis, which was also detected by MDCT. MDCT showed no significant stenosis in 25 of 27 grafts without a severe lesion (specificity 93\%, positive predictive value $89 \%$, negative predictive value $100 \%$ ).

\section{Lesion detection in native vessels}

The reference standard ICA detected 359 lesions with a diameter stenosis $>50 \%$ and MDCT detected 304 of 359 $(85 \%)$. Twenty nine of 55 (53\%) missed lesions were located in distal segments and side branches. Sensitivity, specificity, and positive and negative predictive values for the per patient analysis were $85 \%, 98 \%, 91 \%$, and $96 \%$, respectively. Table 2 summarises results of the segmental and per vessel analyses.

The correct clinical diagnosis was obtained for 110 of 120 $(92 \%)$ patients. MDCT detected all stenoses in 86 of 120 (72\%) patients.

\section{DISCUSSION}

The new finding of the present study is that the latest improvements of MDCT technology with faster gantry rotation time and submillimetre slices have led to a further

Table 2 Diagnostic accuracy of MDCT in detecting and excluding severe lesions per segment and per vessel

\begin{tabular}{|c|c|c|c|c|c|c|c|c|c|}
\hline & Prevalence & Sensitivity & Specificity & PPV & NPV & TP $(n=304)$ & FP $(n=29)$ & $\mathrm{TN}(\mathrm{n}=1172)$ & $\mathrm{FN}(\mathrm{n}=55)$ \\
\hline \multicolumn{10}{|c|}{ RCA segments } \\
\hline Total & $38 \%$ & $91 \%$ & $95 \%$ & $91 \%$ & $95 \%$ & NA & NA & NA & NA \\
\hline 1 & $21 \%$ & $96 \%$ & $99 \%$ & $96 \%$ & $99 \%$ & 24 & 1 & 94 & 1 \\
\hline 2 & $36 \%$ & $93 \%$ & $99 \%$ & $98 \%$ & $96 \%$ & 40 & 1 & 76 & 3 \\
\hline 3 & $28 \%$ & $94 \%$ & $99 \%$ & $97 \%$ & $98 \%$ & 32 & 1 & 85 & 2 \\
\hline 4 & $18 \%$ & $90 \%$ & $97 \%$ & $86 \%$ & $98 \%$ & 19 & 3 & 96 & 2 \\
\hline \multicolumn{10}{|c|}{ LM segment } \\
\hline 5 & $7 \%$ & $88 \%$ & $99 \%$ & $88 \%$ & $99 \%$ & 7 & 1 & 111 & 1 \\
\hline \multicolumn{10}{|c|}{ LAD segments } \\
\hline Total & $56 \%$ & $78 \%$ & $87 \%$ & $88 \%$ & $75 \%$ & NA & NA & NA & NA \\
\hline 6 & $21 \%$ & $84 \%$ & $96 \%$ & $84 \%$ & $96 \%$ & 21 & 4 & 91 & 4 \\
\hline 7 & $38 \%$ & $89 \%$ & $97 \%$ & $95 \%$ & $94 \%$ & 41 & 2 & 72 & 5 \\
\hline 8 & $21 \%$ & $80 \%$ & $100 \%$ & $100 \%$ & $95 \%$ & 20 & 0 & 95 & 5 \\
\hline 9 & $23 \%$ & $67 \%$ & $94 \%$ & $75 \%$ & $91 \%$ & 18 & 6 & 87 & 9 \\
\hline 10 & $19 \%$ & $39 \%$ & $96 \%$ & $69 \%$ & $87 \%$ & 9 & 4 & 93 & 14 \\
\hline \multicolumn{10}{|c|}{ LCx segments } \\
\hline Total & $42 \%$ & $91 \%$ & $95 \%$ & $91 \%$ & $95 \%$ & NA & NA & NA & NA \\
\hline 11 & $20 \%$ & $92 \%$ & $97 \%$ & $88 \%$ & $98 \%$ & 22 & 3 & 93 & 2 \\
\hline 12 & $25 \%$ & $90 \%$ & $99 \%$ & $96 \%$ & $97 \%$ & 27 & 1 & 89 & 3 \\
\hline 13 & $24 \%$ & $86 \%$ & $98 \%$ & $92 \%$ & $96 \%$ & 24 & 2 & 90 & 4 \\
\hline
\end{tabular}

FN, false negative; FP, false positive; NA, not applicable; NPV, negative predictive value; PPV, positive predictive value; TN, true negative; TP, true positive. 
stabilisation of image quality. In our study evaluating the entire coronary tree, only $7 \%$ of coronary segments, the majority in side branches and distal vessel segments, were not analysable.

\section{Image quality and lesion detection}

Our study of a large patient population reproduced the previously reported promising results on lesion detection. There are, however, important differences between these studies and ours, which underline the improved diagnostic accuracy of the latest MDCT generation. Nieman et al ${ }^{1}$ and Ropers $^{2}$ et al reported on highly selected populations with clinical suspicion of CAD, but they studied only vessel segments with a diameter $>2.0 \mathrm{~mm}$ and $>1.5 \mathrm{~mm}$, respectively. In contrast, we investigated consecutive patients who been scheduled for ICA, and all vessel segments were included. Even patients with advanced CAD were studied by MDCT, which is highlighted by the relatively high mean calcium mass. In addition, although all patients received $\beta$ blockade as standard medication or immediately before the MDCT, there was a wide range of heart rates during scanning. Despite these factors, which are known to influence image quality of MDCT, the number of segments with insufficient image quality was low. However, as table 2 shows, vessel size and location still had a significant impact on the diagnostic accuracy of MDCT. This is also underlined by the very good accuracy when evaluating bypass grafts.

Most lesions missed were located in diagonal, marginal, and posterolateral branches. Although temporal resolution has been improved and spatial resolution is now $0.5 \times 0.6 \times 0.6 \mathrm{~mm}$, especially smaller segments $(<1.5$ diameter) are still susceptible to motion artefacts and even severe lesions may be obscured. The accuracy in larger segments was, however, consistently higher than $90 \%$.

\section{Clinical use of MDCT}

The correct clinical diagnosis, which is the most relevant information in clinical practice when looking at the individual patient, defined in our study as the detection of at least one significant lesion, was obtained for $92 \%$ of patients. A complete correspondence with the reference standard ICA was found in $72 \%$ of patients. These data suggest that, although we documented a further improvement of diagnostic accuracy, the latest MDCT generation is not ready to replace diagnostic ICA. As table 2 indicates, one significant left main stenosis as well as several lesions located within proximal vessel segments were not accurately classified by MDCT. All lesions were, however, detected but lesion severity was underestimated. This was mainly due to severe calcifications, which are well known to prohibit accurate determination of the vessel lumen. ${ }^{3}$ Thus, in case of severe calcifications or motion artefacts, the diagnostic accuracy of MDCT is insecure and ICA should be used for clarification in these patients.

The consistently high negative predictive value, which was also documented by other studies, ${ }^{1245}$ underlines, however, that in the case of good image quality and absence of relevant calcifications or motion artefacts, MDCT may be useful for the exclusion of significant lesions in well defined patient groups, which have to be outlined by future studies.

Limitations of MDCT remain radiation exposure (4-6 mSv $)^{6}$ and the need for iodinated contrast media. Direct comparison with ICA may, however, be misleading, since MDCT as a cross sectional technique allows evaluation not only of the vessel lumen but also of the vessel wall and plaque morphology. ${ }^{78}$

\section{Study limitations}

Although the number of patients was relatively high $(\mathrm{n}=124)$ and the MDCT investigators were blinded to the ICA results, this was a single centre trial. Further multicentre studies are required to confirm our data. Stented lesions were excluded from analysis, since current technology does not allow accurate visualisation and evaluation of the vessel lumen within the stents. This indication has to be addressed by further studies with improved technology in the future.

Furthermore, the present study was not designed to evaluate MDCT in selected groups of patients. Since our objective was to investigate image quality and diagnostic accuracy in consecutive patients scheduled for ICA, this question has to be addressed by further studies. A limitation remains that although the initial success of recruitment was high (178 of $195(91 \%))$, only $64 \%$ of patients actually were suitable for participation in our study.

\section{Conclusions}

Compared with magnetic resonance imaging and electron beam computed tomography, the latest MDCT appears to be the most advanced non-invasive imaging modality to visualise human coronary arteries. However, further improvements of spatial and temporal resolutions are still required to challenge diagnostic ICA. ${ }^{9} 10$

\section{Authors' affiliations}

A Kuettner, T Drosch, M Heuschmid, C D Claussen, A F Kopp, Department for Radiology, Division of Diagnostic Radiology, EberhardKarls University of Tuebingen, Tuebingen, Germany

T Beck, K Kettering, C Burgstahler, S Schroeder, Department for Internal Medicine, Division of Cardiology, Eberhard-Karls University of Tuebingen

\section{REFERENCES}

1 Nieman K, Cademartiri F, Lemos PA, et al. Reliable noninvasive coronary angiography with fast submillimeter multislice spiral computed tomography. Circulation 2002;106:2051-4.

2 Ropers D, Baum U, Pohle K, et al. Detection of coronary artery stenoses with thin-slice multi-detector row spiral computed tomography and multiplanar reconstruction. Circulation 2003;107:664-6.

3 Kuettner A, Kopp AF, Schroeder S, et al. Diagnostic accuracy of multidetector computed tomography coronary angiography in patients with angiographically proven coronary artery disease. J Am Coll Cardiol 2004;43:831-9.

4 Martuscelli E, Romagnoli A, D'Eliseo A, et al. Accuracy of thin-slice computed tomography in the detection of coronary stenoses. Eur Heart $J$ 2004;25:1043-8

5 Mollet NR, Cademartiri F, Nieman K, et al. Multislice spiral computed tomography coronary angiography in patients with stable angina pectoris. J Am Coll Cardiol 2004; 43:2265-70.

6 Jakobs TF, Becker CR, Ohnesorge B, et al. Multislice helical CT of the heart with retrospective ECG gating: reduction of radiation exposure by ECGcontrolled tube current modulation. Eur Radiol 2002;12:1081-6.

7 Achenbach S, Moselewski F, Ropers D, et al. Detection of calcified and noncalcified coronary atherosclerotic plaque by contrast-enhanced, submillimeter multidetector spiral computed tomography: a segment-based comparison with intravascular ultrasound. Circulation 2004;109:14-7.

8 Leber AW, Knez A, Becker A, et al. Accuracy of multidetector spiral computed tomography in identifying and differentiating the composition of coronary atherosclerotic plaques: a comparative study with intracoronary ultrasound. J Am Coll Cardiol 2004;43:1241-7.

9 Achenbach S. Detection of coronary stenoses by multidetector computed tomography: it's all about resolution. J Am Coll Cardiol 2004;43:840-1.

10 Budoff MJ, Achenbach S, Duerinckx A. Clinical utility of computed tomography and magnetic resonance techniques for noninvasive coronary angiography. J Am Coll Cardiol 2003;42:1867-78. 